PROCEEDINGS OF THE

AMERICAN MATHEMATICAL SOCIETY

Volume 126, Number 1, January 1998, Pages 105-113

S 0002-9939(98)04357-3

\title{
MICROLOCAL ANALYSIS OF ULTRADISTRIBUTIONS
}

\author{
STEVAN PILIPOVIĆ
}

(Communicated by Palle E. T. Jorgensen)

\begin{abstract}
The ultradistributional wave front sets of an ultradistribution $u$ are characterized by the behaviour of $K * u$ on the boundary of the tube domain $D \mathbf{R}^{n}$, where $K$ is the kernel analysed by Hörmander.
\end{abstract}

\section{INTRODUCTION}

We follow the results and ideas of Chapter 8 in [2] and characterize the ultradistributional wave front sets of type $W F^{*}$ and $W F_{*}$ for a tempered ultradistribution $u$ by using its convolution with the kernel $K$ (cf. [2]). We give the assertions which are analogous to the corresponding ones for distributions.

The paper is organized as follows. In Section 2 we recall the definitions of ultradistributional wave front sets $W F^{*}$ and $W F_{*}$. These notions are introduced by Eida [1] and Komatsu [4] and called singular spectrums $S S^{*}$ and $S S_{*}$. In Section 3 we recall the definition of tempered ultradistribution spaces $\mathcal{S}^{\prime *}$ (cf. [5], [7]) and a theorem from [8] which is needed for later use. In Section 4 we give the microlocal analysis of a $u \in \mathcal{S}^{\prime *}$ (Theorems 2, 3 and Corollary 1). Necessary and sufficient conditions for an analytic function in the tube domain $D \mathbf{R}^{n}$ which determine its boundary value in $\mathcal{S}^{\prime *}$ are given in Theorem 2 .

\section{NOTATION AND NOTIONS}

By $M_{p}, p \in \mathbf{N}_{0}$, we denote a sequence of positive numbers with $M_{0}=1$. We refer to [3] and [6] for the meaning of conditions (M.1), (M.2)', (M.2), (M.3)' and (M.3). We also use the following condition ([6]):

$(\mathrm{M} .1)^{*} \quad M_{p-1}^{*} M_{p+1}^{*} \leq M_{p}^{*}, p \in \mathbf{N}$, where $M_{0}^{*}=1, M_{p}^{*}=M_{p} / p !, p \in \mathbf{N}$.

Let $M_{p}$ satisfy (M.1) and (M.3)'. The associated function $M(\rho)$ and the growth function $\tilde{M}(\rho)$ related to $M_{p}$ are defined by

$$
M(\rho)=\sup _{p \in \mathbf{N}_{0}} \ln \frac{\rho^{p}}{M_{p}}, \tilde{M}(\rho)=\sup _{p \in \mathbf{N}_{0}} \ln \frac{\rho^{p}}{M_{p}^{*}}, \rho>0 .
$$

Note, for a given $L>0$ there is $L_{1}>0$ such that ([8])

$$
M(L|\xi|)-|\eta||\xi| \leq \tilde{M}\left(L_{1} /|\eta|\right), \quad \xi, \eta \in \mathbf{R}^{n} .
$$

Received by the editors February 27, 1996.

1991 Mathematics Subject Classification. Primary 32A40, 46F15; Secondary 58G07.

Key words and phrases. Ultradistributions, wave front.

(c) 1998 American Mathematical Society 
We denote by $\Omega$ an open set in $\mathbf{R}^{n}$, and $K \subset \subset \Omega$ means that $K$ is a compact subset of $\Omega$. Recall,

$$
\|\varphi\|_{K, h, M_{p}}=\sup _{x \in K, \alpha \in \mathbf{N}_{0}^{n}} \frac{\left|\varphi^{(\alpha)}(x)\right|}{h^{|\alpha|} M_{|\alpha|}}, \varphi \in C^{\infty}(\Omega) .
$$

The symbol $*$ is used for both $\left(M_{p}\right)$ and $\left\{M_{p}\right\}$. We refer to [3] for the definitions of $\mathcal{D}^{*}(\Omega), \mathcal{D}_{K}^{*}(\Omega)$ and $\mathcal{D}_{K}^{\prime *}(\Omega)$. Throughout the paper we will assume that (M.1), $(\mathrm{M} .2)^{\prime}$ and (M.3) hold. Eida [1] and Komatsu [4] have defined $S S_{*^{-}}$and $S S^{*^{*}}$ singular support of a hyperfunction $f$. We will recall the definitions related to ultradistributions and call them wave front sets.

Let $N_{p}$ be a sequence of positive numbers which satisfies (M.1), (M.2)', (M.3)' and $N_{0}=1$. Then $([3])$ :

$$
\left(N_{p}\right) \leq\left(M_{p}\right) \quad\left(\text { resp., }\left\{N_{p}\right\} \leq\left\{M_{p}\right\}\right)
$$

if there are constants $L>0$ and $C>0$ (resp., for every $\epsilon>0$ there is $C_{\epsilon}>0$ ) such that

$$
\left.N_{p} \leq C L^{p} M_{p} \quad \text { (resp., } \quad N_{p} \leq C_{\epsilon} \epsilon^{p} M_{p}\right), \quad p \in \mathbf{N}_{0} .
$$

Let $f \in \mathcal{D}^{\prime \dagger}$, where $\dagger=N_{p} \leq *=M_{p}$. Then $(x, \omega) \in S^{*} \Omega=\Omega \times S^{n-1}$ is not in $W F_{*} f$ (resp., not in $W F^{*} f$ ) iff there exist a neighborhood $U \subset \Omega$ of $x$ and a conic neighborhood $\Gamma$ of $\omega$ of the form $\Gamma=\{\xi \neq 0 ;|\xi /| \xi|-\omega|<\eta\}$ such that for every $\phi \in \mathcal{D}^{*}(U)$ the following hold:

In the $\left(M_{p}\right)$ case, for every $\epsilon>0$ there is $C_{\epsilon}>0$ such that $|\widehat{\phi f}(\xi)| \leq C_{\epsilon} e^{-M(\epsilon|\xi|)}$, $\xi \in \Gamma$ (resp., there are $k>0$ and $C>0$ such that $|\widehat{\phi f}(\xi)| \leq C e^{M(k|\xi|)}, \xi \in \Gamma$ ).

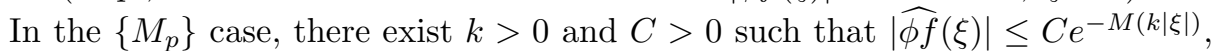
$\xi \in \Gamma$ (resp., for every $\epsilon>0$, there is $C_{\epsilon}>0$ such that $|\widehat{\phi f}(\xi)| \leq C_{\epsilon} e^{M(\epsilon|\xi|)}, \xi \in \Gamma$ ). Note, the notion $W F_{\left\{M_{p}\right\}}$ is equal to Hörmander's notion $W F_{L}$.

The definition of the singular spectrum $S S f$, where $f \in \mathcal{B}(\Omega)$, is given by Sato (cf. [10]). For an $f \in \mathcal{D}^{\prime *}(\Omega),(x, \omega) \in S^{*} \Omega$ is not in $S S f$ if this point is not in $S S\{f\}$, where $\{f\}$ denotes the corresponding hyperfunction. This notion is equal to Hörmander's $W F_{A} f$, the analytic wave front set of $f$ ([2], Definition 9.3.2, Theorem 9.6.3) and we will use this notation.

We also use the definition according to which $(x, \xi) \in \Omega \times\left(\mathbf{R}^{n} \backslash\{0\}\right)$ is an element of the corresponding singular spectrum defined above if this holds for $(x, \xi /|\xi|)$.

Let $D \mathbf{R}^{n}=\left\{z \in \mathbf{C}^{n} ;|\operatorname{Im} z|<1\right\}$ and $S^{*} \mathbf{R}^{n}=\partial D \mathbf{R}^{n}$. Recall ([4]), $\left.O^{*}\right|_{D \mathbf{R}^{n}}$ (resp., $\left.O_{*}\right|_{D \mathbf{R}^{n}}$ ) is a sheaf over $\mathbf{C}^{n}$ of holomorphic functions in $D \mathbf{R}^{n}$ which satisfy the following growth condition near $S^{*} \mathbf{R}^{n}$ :

Let $U$ be an open set in $\mathbf{C}^{n}$. Then a function $F(z)$ is in $\left.O^{*}\right|_{D \mathbf{R}^{n}}(U)$ (resp., $\left.\left.O_{*}\right|_{D \mathbf{R}^{n}}(U)\right)$ if $F$ is holomorphic in $D \mathbf{R}^{n} \cap U$ such that for every compact set $K \subset \subset U$,

in the $\left(M_{p}\right)$ case, there are $C>0$ and $k>0$,

in the $\left\{M_{p}\right\}$ case, for every $k>0$ there is $C>0$ such that

$$
|F(z)| \leq C e^{\tilde{M}\left(\frac{k}{1-|y|}\right)}, \quad z=x+\sqrt{-1} y \in K \cap D \mathbf{R}^{n}
$$

(resp., for every compact set $K \subset \subset U$,

in the $\left(M_{p}\right)$ case, for every ultradifferential operator $P(\partial)$ of class $\left(M_{p}\right)$, in the $\left\{M_{p}\right\}$ case, for every ultradifferential operator $P(\partial)$ of class $\left\{M_{p}\right\}$

$$
P\left(\partial_{z}\right) F \quad \text { is bounded in } K \cap D \mathbf{R}^{n} \text {.) }
$$




\section{TEMPERED Ultradistributions}

We will recall the definitions and the basic structural properties of tempered ultradistributions (cf. [5] and [7]). Let $m>0$. The space of smooth functions $\varphi$ on $\mathbf{R}^{n}$ which satisfy

$$
\sigma_{m, 2}(\varphi)=\left(\sum_{\alpha, \beta \in \mathbf{N}_{0}^{n}} \int_{\mathbf{R}^{n}}\left|\frac{m^{|\alpha+\beta|}}{M_{|\alpha|} M_{|\beta|}}\left(1+|x|^{2}\right)^{|\beta| / 2} \varphi^{(\alpha)}(x)\right|^{2} d x\right)^{1 / 2}<\infty,
$$

equipped with the topology induced by the norm $\sigma_{m, 2}$, is denoted by $\mathcal{S}_{2}^{M_{p}, m}$. The strong duals of $\mathcal{S}^{\left(M_{p}\right)}=\operatorname{proj} \lim _{m \rightarrow \infty} \mathcal{S}_{2}^{M_{p}, m}$ and $\mathcal{S}^{\left\{M_{p}\right\}}=$ ind $\lim _{m \rightarrow 0} \mathcal{S}_{2}^{M_{p}, m}$ are called spaces of tempered ultradistributions of Beurling and Roumieau type. For every fixed $p \in[1, \infty]$, the family of norms $\left\{\sigma_{m, 2} ; m>0\right\}$ is equivalent to the family of norms $\left\{\sigma_{m, p} ; m>0\right\}$ where instead of the $L^{2}$ norm we put the $L^{p}$ norm. In fact, in the sequel we will use the family of norms

$$
s_{h}(\phi)=\sup \left\{\frac{h^{|\alpha|}}{M_{|\alpha|}}\left|\phi^{(\alpha)}(x)\right| e^{M(h|x|)} ; \alpha \in \mathbf{N}_{0}^{n}, x \in \mathbf{R}^{n}\right\}, \quad h>0,
$$

which is equivalent to $\left\{\sigma_{h, 2} ; h>0\right\}$.

$\mathcal{S}^{\left(M_{p}\right)}$ and $\mathcal{S}^{\left\{M_{p}\right\}}$ are $(F S)$ - and $(L S)$-spaces respectively. If (M.2) holds, they are $(F N)$ - and $(L N)$-spaces respectively and $\mathcal{D}^{*} \hookrightarrow \mathcal{S}^{*} \hookrightarrow \mathcal{E}^{*}, \quad \mathcal{S}^{*} \hookrightarrow \mathcal{S}$, where " $A \hookrightarrow B$ " means that $A$ is dense in $B$ and the inclusion mapping is continuous.

An $f \in \mathcal{D}^{\prime *}$ is in $\mathcal{S}^{\prime *}$ if and only if there exists a family $F_{\alpha, \beta}, \alpha, \beta \in \mathbf{N}_{0}^{n}$, in $L^{2}\left(\mathbf{R}^{n}\right)$ such that

$$
f=\sum_{\alpha, \beta \in \mathbf{N}_{0}^{n}}\left(\left(1+|x|^{2}\right)^{\beta / 2} F_{\alpha, \beta}\right)^{(\alpha)} \text { in } \mathcal{S}^{*}
$$

and in the case $\mathcal{S}^{\prime\left(M_{p}\right)}$, there exists $k>0$ (resp., in the case $\mathcal{S}^{\prime\left\{M_{p}\right\}}$, for every $k>0$ ) such that

$$
\left(\sum_{\alpha, \beta \in \mathbf{N}_{0}^{n}} \int_{\mathbf{R}^{n}}\left|\frac{M_{|\alpha|} M_{|\beta|}}{k^{|\alpha+\beta|}} F_{\alpha, \beta}(x)\right|^{2}\right)^{1 / 2}<\infty .
$$

The Fourier transformation is an isomorphism of $\mathcal{S}^{*}$ onto itself.

We recall the next theorem for later use.

Theorem 1 ([8]). Assume (M.1)* , (M.2) and (M.3)' hold for $\dagger=N_{p}\left(N_{0}=1\right)$. Let $\Gamma$ be an open convex cone in $\mathbf{R}^{n}$ and $F$ be an analytic function in

$$
Z=\left\{z \in \mathbf{C}^{n} ; \operatorname{Im} z \in \Gamma,|\operatorname{Im} z|<\gamma\right\}
$$

for some $\gamma>0$. Moreover, assume

$$
|F(x+\sqrt{-1} y)| \leq C_{a, b} e^{N(a|x|)+\tilde{N}\left(\frac{b}{|y|}\right)}, \quad x+\sqrt{-1} y \in Z,
$$

in the $\left(N_{p}\right)$ case for some $a>0, b>0$, and $C_{a, b}>0$, and in the $\left\{N_{p}\right\}$ case for every $a>0, b>0$ there exists $C_{a, b}>0$. Then

$$
F(x+\sqrt{-1} y) \stackrel{\mathcal{S}^{\dagger \dagger}}{\rightarrow} F(x+\sqrt{-1} 0), \quad y \rightarrow 0, \quad y \in \Gamma .
$$


Note $([8])$,

$$
\begin{gathered}
\langle F(x+\sqrt{-1} 0), \varphi(x)\rangle=\int_{\mathbf{R}^{n}} F(x+\sqrt{-1} Y) \Phi(x+\sqrt{-1} Y) d x \\
+2 \sqrt{-1} \sum_{i=1}^{n} Y_{i} \int_{0}^{1} \int_{\mathbf{R}^{n}} \frac{\partial}{\partial \bar{z}_{i}} \Phi(x+\sqrt{-1} t Y) F(x+\sqrt{-1} Y t) d t d x .
\end{gathered}
$$

Moreover, there exists $C>0$ such that

$$
|\langle F(x+\sqrt{-1} 0), \varphi(x)\rangle| \leq C s_{h}(\varphi), \varphi \in \mathcal{S}^{\dagger},
$$

where $\Phi(z)$ is the almost analytic extension of $\varphi$ (cf. [6] or [8]).

\section{Microlocal analysis of Ultradistributions}

As in [2], put

$$
I(\xi)=\int_{|\omega|=1} e^{-\langle\omega, \xi\rangle} d \omega, \xi \in \mathbf{R}^{n}, K(z)=(2 \pi)^{-n} \int \frac{e^{\sqrt{-1}\langle z, \xi\rangle}}{I(\xi)} d \xi, z \in D \mathbf{R}^{n} .
$$

We call $K$ Hörmander's kernel. Recall ([2], Lemma 8.4.10) that $K(z)$ is an analytic function in $\tilde{\Omega}=\left\{z \in \mathbf{C}^{n} ;\langle z, z\rangle \notin(-\infty,-1]\right\}$. The properties of this function given in the quoted lemma imply

$$
|K(x+\sqrt{-1} y)| \leq \frac{C n ! e^{-c|x|}}{(1-|y|)^{n}}, \quad z \in D \mathbf{R}^{n},
$$

for some $C>0$ and $c>0$. By using the Cauchy formula on the contour

$$
\Gamma_{t+\sqrt{-1} y}=\Gamma_{t_{1}+\sqrt{-1} y_{1}} \times \cdots \times \Gamma_{t_{n}+\sqrt{-1} y_{n}},
$$

where $\Gamma_{t_{j}+\sqrt{-1} y_{j}}$ is the boundary of

$$
\left\{\left(\tau_{j}, u_{j}\right) ;\left|t_{j}-\tau_{j}\right|<1,\left|y_{j}-u_{j}\right|<(1-|y|) / 2\right\},
$$

it follows that for some $C>0$ and $c>0$

$$
\left|\frac{\partial^{\alpha}}{\partial t^{\alpha}} K(t+\sqrt{-1} y)\right|<C \frac{\alpha ! n !}{(1-|y|)^{|\alpha|+n}} e^{-c|t|}, t \in \mathbf{R}^{n},|y|<1, \quad \alpha \in \mathbf{N}_{0}^{n} .
$$

This implies that $K(\cdot+\sqrt{-1} y) \in \mathcal{S}^{*}$ for every fixed $y,|y|<1$.

The next theorem is proved in [2] for tempered distributions.

Theorem 2. Let $\dagger=N_{p}$ and $*=M_{p}$ satisfy (M.1) ${ }^{*}$, (M.2) and (M.3)' and let $N_{p} \leq M_{p}$. Let $u \in \mathcal{S}^{\dagger}$ and $U(z)=(u * K)(z)=\langle u(t), K(x-t+\sqrt{-1} y)\rangle, z \in D \mathbf{R}^{n}$.

a) Then $U$ is analytic in $D \mathbf{R}^{n}$ and

in the $\left(N_{p}\right)$ case, for some $a>0, b>0$ there is $C_{a, b}>0$,

in the $\left\{N_{p}\right\}$ case, for every $a>0, b>0$ there is $C_{a, b}>0$, such that

$$
|U(z)| \leq C_{a, b} e^{N(a|x|)+\tilde{N}(b /(1-|y|))}, z=x+\sqrt{-1} y \in D \mathbf{R}^{n} .
$$

For every $\omega \in S^{n-1}$ there exists the limit

$$
\lim _{\substack{y \rightarrow \omega \\ z \in D \mathbf{R}^{n}}}\langle U(x+\sqrt{-1} y), \phi(x)\rangle=\langle U(x+\sqrt{-1} \omega), \phi(x)\rangle, \phi \in \mathcal{S}^{\dagger},
$$

and the mapping $S^{n-1} \ni \omega \mapsto U(\cdot+\sqrt{-1} \omega) \in{\mathcal{\mathcal { S } ^ { \prime }}}^{\dagger}$ is continuous. Moreover,

$$
\langle u, \phi\rangle=\int_{\mathbf{R}^{n}}\langle U(\cdot+\sqrt{-1} \omega), \phi\rangle d \omega, \phi \in \mathcal{S}^{\dagger} .
$$


b) Conversely, if $U$ is given satisfying (4), then (5) defines an ultradistribution in $\mathcal{S}^{\dagger}$.

c1) $q=\left(x_{0}, \omega_{0}\right) \notin W F^{*} u$ if and only if $U$ is $\mathcal{O}^{*}$ in a neighborhood of $x_{0}-\sqrt{-1} \omega_{0}$.

c2) $q \notin W F_{*} u$ if and only if $U$ is $\mathcal{O}_{*}$ in a neighborhood of $x_{0}-\sqrt{-1} \omega_{0}$.

c3) $q \notin W F_{A} u$ if and only if $U$ is analytic at $x_{0}-\sqrt{-1} \omega_{0}$ (i.e. in a neighborhood of this point).

Proof. a) We will give only the proof in the $\left(M_{p}\right)$ case. There exist $h>0, C_{h}>0$ and suitable constants which do not depend on $h$, such that

$$
\begin{aligned}
|\langle u(t), K(z-t)\rangle| & \leq C_{h} \sup _{\substack{\alpha \in \mathbf{N}^{n} \\
t \in \mathbf{R}^{n}}}\left\{\frac{h^{\alpha}\left|\frac{\partial^{\alpha}}{\partial t^{\alpha}} K(z-t)\right| e^{N(h|t|)}}{N_{|\alpha|}}\right\} \\
& \leq C C_{h} \sup _{\substack{\alpha \in \mathbf{N}_{0}^{n} \\
t \in \mathbf{R}^{n}}}\left\{\frac{h^{\alpha} \alpha ! n ! e^{-c|x-t|}}{N_{|\alpha|}(1-|y|)^{|\alpha|+n}} e^{N(h|t|)}\right\} \\
& \leq C_{1} C_{h} e^{N(h|x|)} \sup _{\alpha \in \mathbf{N}_{0}^{n}} \frac{h^{\alpha} \alpha !}{(1-|y|)^{|\alpha|} N_{|\alpha|}} \\
& \leq C_{1} C_{h} e^{N(h|x|)+\tilde{N}\left(\frac{h}{1-|y|}\right)}, z \in D \mathbf{R}^{n} .
\end{aligned}
$$

This proves (4). Since the Fourier transformation of $U(\cdot+\sqrt{-1} y),|y|<1$, is $e^{-\langle y, \xi\rangle} \hat{u}(\xi) / I(\xi), \xi \in \mathbf{R}^{n}$, and

$$
I(\xi)=(2 \pi)^{(n-1) / 2} \frac{e^{|\xi|}}{|\xi|^{(n-1) / 2}}(1+\mathcal{O}(1 /|\xi|)),|\xi| \rightarrow \infty
$$

([2], p. 287), it follows that $y \mapsto U(\cdot+\sqrt{-1} y),|y| \leq 1$, is a continuous function $\{y ;|y| \leq 1\} \rightarrow \mathcal{S}^{\left(N_{p}\right)}$.

Let us prove this. For any $\phi \in \mathcal{S}^{\dagger}, \xi \in \mathbf{R}^{n}, \alpha \in \mathbf{N}_{0}$ and $|y| \leq 1$ we have

$$
\begin{gathered}
\left|\left(e^{-\langle y, \xi\rangle}(1 / I(\xi)) \phi(\xi)\right)^{(\alpha)}\right| \\
=\left|\sum_{i \leq \alpha}\left(\begin{array}{c}
\alpha \\
i
\end{array}\right)(y)^{\alpha-i} e^{-\langle y, \xi\rangle} \sum_{p \leq i}\left(\begin{array}{l}
i \\
p
\end{array}\right) \phi^{(i-p)}(\xi)(1 / I(\xi))^{(p)}\right| .
\end{gathered}
$$

Since $\left|(I(\xi))^{(\beta)}\right| \leq I(\xi), \quad \xi \in \mathbf{R}^{n}, \beta \in \mathbf{N}_{0}^{n}$, we conclude

$$
\left|(1 / I(\xi))^{(p)}\right| \leq 2^{|p|} p ! / I(\xi), \quad \xi \in \mathbf{R}^{n} .
$$

Now by (6),

$$
\left|\left(e^{-\langle y, \xi\rangle}(1 / I(\xi)) \phi(\xi)\right)^{(\alpha)}\right| \leq C 3^{|\alpha|}(1+|\xi|)^{(n-1) / 2} \sup _{|i| \leq \alpha}\left|\phi^{(i)}(\xi)\right|
$$

which implies:

$$
\begin{gathered}
\langle u, \phi\rangle=\lim _{r \rightarrow 1}\left\langle\int_{|\omega|=1} \frac{e^{\langle-r \omega, \xi\rangle} \hat{u}(\xi)}{I(\xi)} d \omega, \hat{\phi}(\xi)\right\rangle \\
=\lim _{r \rightarrow 1} \int_{|\omega|=1}\left\langle\frac{e^{-r\langle\omega, \xi\rangle} \hat{u}(\xi)}{I(\xi)}, \hat{\phi}(\xi)\right\rangle d \omega=\int_{|\omega|=1}\langle U(x+\sqrt{-1} \omega), \phi(x)\rangle d \omega,
\end{gathered}
$$

where $y=r \omega, \omega \in S^{n-1}$. 
b) Conversely, if $U$ is analytic in $D \mathbf{R}^{n}$ and satisfies (4), then Theorem 1 , more precisely (2) and (3), imply that

$$
\lim _{\substack{y \rightarrow \omega \\ z \in D \mathbf{R}^{n}}} U_{y}=U_{\omega}, \quad\left|\left(\omega_{1}, \ldots, \omega_{n}\right)\right|=1
$$

exists in $\mathcal{S}^{\prime\left(N_{p}\right)}$, where $U_{y}=U(\cdot+\sqrt{-1} y),|y|<1$ and

$$
\begin{gathered}
\left\langle U_{\omega}, \phi\right\rangle=\int U(x) \Phi(x-\sqrt{-1} \omega) d x \\
-2 \sqrt{-1} \sum \omega_{i} \int_{0}^{1} \int_{\mathbf{R}^{n}} U(x+\sqrt{-1}(1-t) \omega) \frac{\partial \Phi}{\partial \bar{z}_{1}}(x+\sqrt{-1}(1-t) \omega) d t d x .
\end{gathered}
$$

In fact, we have to put $U(z+\sqrt{-1} \omega)=F(z), Y=-\omega$, and the conclusion follows as in Theorem 1.

Moreover, it follows that $S^{n-1} \ni \omega \mapsto U_{\omega} \in \mathcal{S}^{\prime\left(N_{p}\right)}$ is continuous, which implies that by

$$
\phi \mapsto\langle u, \phi\rangle=\int\left\langle U_{\omega}(x), \phi(x)\right\rangle d \omega, \phi \in \mathcal{S}^{\left(M_{p}\right)},
$$

an element from $\mathcal{S}^{\prime\left(N_{p}\right)}$ is defined. One can easily show that $U=u * K$.

c) We will prove the "if" parts.

Suppose $q \notin W F^{*} u$. Let $\varphi \in \mathcal{D}^{*}(U)$, where $U=\left\{x ;\left|x-x_{0}\right|<2 r\right\}$ and $0 \leq \varphi \leq 1, \varphi \equiv 1$ on $\left\{x ;\left|x-x_{0}\right|<r\right\}$.

Note that

$$
|\widehat{\varphi u}(\xi)|<C_{1} e^{M\left(L_{1}|\xi|\right)}, \quad \xi \in \mathbf{R}^{n},
$$

for some $L_{1}>0$ and some $C_{1}>0$ in the $\left(M_{p}\right)$ case (resp., for every $L_{1}>0$ there is $C_{1}>0$ in the $\left\{M_{p}\right\}$ case). Put $U=\varphi u * K+(1-\varphi) u * K=U_{1}+U_{2}$. This decomposition is also used in parts b) and c). Let $x \in\left\{x ;\left|x-x_{0}\right|<r / 2\right\}$. Then,

$$
(1-\varphi(t)) K(x+\sqrt{-1} y-t)=0, \quad\left|t-x_{0}\right|<r, \quad x+\sqrt{-1} y \in \mathbf{C}^{n} .
$$

If $\left|t-x_{0}\right| \geq r$, then $|x-t| \geq r / 2$ and for $|y|<1+r / 2$,

$$
t \mapsto K(x+\sqrt{-1} y-t)
$$

is a function which belongs to $S^{*}$.

This implies that

$$
U_{2}(z)=\langle u(t),(1-\varphi(t)), K(z-t)\rangle, \quad z \in D \mathbf{R}^{n},
$$

is analytic in

$$
\left\{x+\sqrt{-1} y ;|y|<1+r / 2,\left|x-x_{0}\right|<r / 2\right\},
$$

which is a neighborhood of $x_{0}-\sqrt{-1} \omega_{0}$. So in the proofs of c1), c2) and c3) below we have to consider $U_{1}$.

c1) Let $\gamma$ be an open convex cone which contains $\omega_{0}, \epsilon>0$ and $\left|y+\omega_{0}\right|<\epsilon$, $|y|<1$. We shall use the following inequalities:

$$
M(L|\xi|)-\langle\xi, y\rangle-|\xi| \leq \tilde{M}(\tilde{L} /(1-|y|)), \quad \xi \in \gamma
$$

which holds for some $\tilde{L}>0$ (see $(1))$, and

$$
-\langle y, \xi\rangle-|\xi| \leq-\epsilon|\xi|, \quad \xi \notin \gamma,
$$

which holds if $\epsilon$ is small enough. 
Let $\left|y+\omega_{0}\right|<\epsilon,|y|<1,\left|x-x_{0}\right|<r$. Then $(z=x+\sqrt{-1} y)$,

$$
\begin{gathered}
\left|U_{1}(z)\right|=|\mathcal{F}(\varphi u * K(z, \cdot))(\xi)| \\
\leq \int_{\mathbf{R}^{n}} \frac{e^{-\langle y, \xi\rangle}}{I(\xi)} \widehat{\varphi u}(\xi) d \xi=\int_{\xi \in \gamma}+\int_{\xi \notin \gamma} .
\end{gathered}
$$

By using (7) and (8) for the first integral and (7) and (9) for the second integral, we obtain that $U_{1}$ is $\mathcal{O}^{*}$ in some neighborhood of $x_{0}-\sqrt{-1} \omega_{0}$.

c2) Let $q \notin W F_{*} u$. By using

$$
\left|P\left(\partial_{z}\right)(u \varphi * K)(z)\right| \leq \int_{\mathbf{R}^{n}} \frac{e^{-\langle y, \xi\rangle}}{I(\xi)} P(\sqrt{-1} \xi) \widehat{u \varphi}(\xi) d \xi,
$$

in a similar way we obtain that for every ultradifferential operator $P\left(\partial_{z}\right)$ of the corresponding class, $P\left(\partial_{z}\right) U$ is bounded in a neighborhood of $x_{0}-\sqrt{-1} \omega_{0}$.

c3) If $\left(x_{0}, \omega_{0}\right) \notin W F_{A} u$, then $\left(x_{0}, \omega_{0}\right) \notin W F_{A} u \phi$. By [2], Definition 9.3.2, it is equivalent to the analyticity of $u \varphi * K$ at $x_{0}-\sqrt{-1} \omega_{0}$.

The converse parts in c1), c2) and c3) follow from the next lemma (see [2], Lemma 8.4, for tempered distributions).

Lemma 1. Let $d \mu$ be a measure on $S^{n-1}$ and $\Gamma$ an open convex cone in $\mathbf{R}^{n}$ such that

$$
\langle y, \omega\rangle<0 \quad \text { if } \quad 0 \neq y \in \bar{\Gamma}, \omega \in \operatorname{supp} d \mu .
$$

Let $U$ be analytic in $D \mathbf{R}^{n}$, and satisfy (4) in $D \mathbf{R}^{n}$. Then

$$
F(z)=\int_{S^{n-1}} U(z+\sqrt{-1} \omega) d \mu(\omega), \operatorname{Im} z \in \Gamma,|\operatorname{Im} z|<\gamma
$$

is analytic and

$$
|F(z)| \leq C_{a, b} e^{N(a|x|)+\tilde{N}(b /(1-|y|))}, \operatorname{Im} z \in \Gamma,|\operatorname{Im} z|<\gamma,
$$

for some $a, b$ and some $C_{a, b}>0$ in the $\left(N_{p}\right)$ case, and for every $a, b$ there is $C_{a, b}>0$ in the $\left\{N_{p}\right\}$ case.

For every measure $d \mu$ on $S^{n-1}$ and

there holds:

$$
U_{\mu}=\int_{S^{n-1}} U(\cdot+\sqrt{-1} \omega) d \mu(\omega),
$$

$W F^{*} U_{\mu} \subset\left\{(x, \omega) ;-\omega \in \operatorname{supp} d \mu\right.$ and $U$ is not $\mathcal{O}^{*}$ at $\left.x-\sqrt{-1} \omega\right\}$.

$W F_{*} U_{\mu} \subset\left\{(x, \omega) ;-\omega \in \operatorname{supp} d \mu\right.$ and $U$ is not $\mathcal{O}_{*}$ at $\left.x-\sqrt{-1} \omega\right\}$.

$W F_{A} U_{\mu} \subset\{(x, \omega) ;-\omega \in \operatorname{supp} d \mu$ and $U$ is not analytic at $x-\sqrt{-1} \omega\}$.

Proof. Let $\omega \in \operatorname{supp} d \mu$ and $\operatorname{Im} z \in \Gamma$. Then for some $C>0$,

$$
1-|\operatorname{Im}(z+\sqrt{-1} \omega)|>\frac{|\operatorname{Im} z|}{2} \text { if }|\operatorname{Im} z|<C \quad([5], \text { Lemma 8.4.12). }
$$

This implies that we may use Theorem 1 for $F$. Thus, $F(\dot{+} \sqrt{-1} \Gamma 0) \in \mathcal{S}^{\prime \dagger}$. Note

$$
W F^{*} U_{\mu} \subset W F_{*} U_{\mu} \subset W F_{A} U_{\mu} \subset \mathbf{R}^{n} \times \Gamma^{0}
$$

where $\Gamma^{0}=\{\xi ;\langle x, \xi\rangle \geq 0$ for every $x \in \Gamma\}$ is the dual cone.

We shall prove only the estimate for $W F^{*} U_{\mu}$ since the other parts can be proved similarly. Denote sing supp* $u=\pi_{1} W F^{*} u$. There holds

$$
\text { sing supp }{ }^{*} U_{\mu} \subset\left\{x ; U \text { is not } \mathcal{O}^{*} \text { at } x+\sqrt{-1} \omega \text { for some } \omega \in \operatorname{supp} d \mu\right\} \text {. }
$$


Decompose

$$
d \mu=\sum_{j=1}^{r} d \mu_{j}, \quad \operatorname{supp} d \mu_{j} \subset \operatorname{supp} d \mu \cap \Gamma_{j},
$$

where $\Gamma_{j}$ are open convex cones such that int $\Gamma_{j}^{0} \neq \emptyset$. By replacing $d \mu$ and $\Gamma$ from the first part of the lemma by $d \mu_{j}$ and $-\operatorname{int}\left(\Gamma_{j}^{0}\right)$ we obtain

$$
\begin{aligned}
& W F^{*} U_{\mu} \quad \subset \bigcup_{j=1}^{r}\left\{(x, \xi) ;-\xi \in \overline{\Gamma_{j}}, U \text { is not } \mathcal{O}^{*}\right. \\
&\text { at } \left.x-\sqrt{-1} \omega \text { for some } \omega \in \Gamma_{j} \cap S^{n-1}\right\} .
\end{aligned}
$$

If $-\frac{\xi}{|\xi|} \notin \operatorname{supp} d \mu$ or $U$ is not $\mathcal{O}^{*}$ at $x-\sqrt{-1} \frac{\xi}{|\xi|}$, there exists the decomposition of $d \mu$ such that $-\xi \notin \overline{\Gamma_{j}}$, for $j=1, \ldots, r$ or $-\xi \notin \overline{\Gamma_{j}}$ for $j=2, \ldots, r$ and $U$ is $\mathcal{O}^{*}$ at $x+\sqrt{-1} \omega$ for every $\omega \in \Gamma_{1} \cap S^{n-1}$. In both cases $(x, \xi) \notin W F^{*} U_{\mu}$.

Immediate consequences of this theorem are the next corollary and theorem (see [2], Corollary 8.4.13 and Theorem 8.4.15 for tempered distributions).

Corollary 1. Let $G_{j}$ be closed subsets of $S^{n-1}$ such that

$\bigcup_{j=1}^{r} G_{j}=S^{n-1}$. Any $u \in \mathcal{S}^{\prime \dagger}\left(\mathbf{R}^{n}\right)$ can be written $u=\sum_{j=1}^{r} u_{j}, u_{j} \in \mathcal{S}^{\prime \dagger}\left(\mathbf{R}^{n}\right)$ $(\dagger \leq *)$ with

a) $W F^{*} u_{j} \subset W F^{*} u \cap \mathbf{R}^{n} \times G_{j}, j=1, \ldots, r$.

b) $W F_{*} u_{j} \subset W F_{*} u \cap \mathbf{R}^{n} \times G_{j}, j=1, \ldots, r$.

c) $W F_{A} u_{j} \subset W F_{A} u \cap \mathbf{R}^{n} \times G_{j}, j=1, \ldots, r$.

If $u \in \mathcal{E}^{\prime \dagger}$, then $u_{j}, j=1, \ldots, r$, have compact supports as well.

If $u=\sum u_{j}^{\prime}$ is another such decomposition, then

$$
\begin{gathered}
u_{j}^{\prime}=u_{j}+\sum_{k} u_{j k}, u_{j k} \in S^{\prime *}, \\
u_{j k}=-u_{j k} \text { and } W F^{*} u_{j k} \subset\left(W F^{*} u\right) \cap \mathbf{R}^{n} \times\left(G_{j} \cap G_{k}\right) .
\end{gathered}
$$

The same holds for $W F_{*} u_{j k}$ and $W F_{A} u_{j k}$.

Theorem 3. Let $\Gamma$ be an open convex cone in $\mathbf{R}^{n}, u \in \mathcal{D}^{\prime *}(\Omega), \Omega \subset \mathbf{R}^{n}$, and $W F_{*} u \subset \Omega \times \Gamma^{0}$. If $\Omega_{1} \subset \subset \Omega$ and $\Gamma_{1}$ is an open convex cone with closure $\subset \Gamma \cup\{0\}$, then there is an $F$ analytic in $\left\{x+\sqrt{-1} y ; x \in \Omega_{1}, y \in \Gamma_{1},|y|<\gamma\right\}$ such that for some $k>0$ and $C>0$ in the $\left(M_{p}\right)$ case (resp., for every $k>0$ there is $C>0$ in the $\left\{M_{p}\right\}$ case $)$,

$$
|F(x+\sqrt{-1} y)| \leq C e^{\tilde{M}(k /|y|)}, x \in \Omega_{1}, y \in \Gamma_{1},|y|<\gamma,
$$

and $F(\cdot+\sqrt{-1} 0)-\left.u\right|_{\Omega_{1}} \in \mathcal{E}^{*}\left(\Omega_{1}\right)$.

\section{REFERENCES}

[1] A. Eida, On the microlocal decomposition of ultradistributions and ultradifferentiable functions, Preprint.

[2] L. Hörmander, The Analysis of Linear Partial Differential Operators I, Springer, Berlin Heidelberg - New York - Tokyo, 1983. MR 91m:35001b

[3] H. Komatsu, Ultradistributions, I - III, J. Fac. Sci. Univ. Tokyo, Sect. IA 20(1973), 25-105; 24(1977) 607 - 628; 29(1982), 653 - 717. MR 47:9277; MR 57:17280; MR 84d:46051

[4] H. Komatsu, Microlocal Analytic in Gevrey Classes and in Convex Domains, Springer, Lec. Not. Math. 1726(1989), 426 - 493.

[5] D. Kovačević, S. Pilipović, Structural properties of the spaces of tempered ultradistributions, Proc. Conf. "Complex Analysis and Generalized Functions", Varna 1991, 169 - 184. MR 95c:46059 
[6] H. J. Petzsche, Generalized functions and the boundary values of holomorphic functions, J. Fac. Sci. Univ. Tokyo, Sect. IA Math. 31 (1984), 391-431. MR 85k:46048

[7] S. Pilipović, Characterization of bounded sets in spaces of ultradistributions, Proc. AMS, 120(1994), 1191-1206. MR 94f:46048

[8] S. Pilipović, Structural Theorems for Ultradistributions, Ann. Polon. Math. 340(1995), 223235. MR 96f: 46076

[9] J. W. de Roewer, Hyperfunctional singular support of distributions, J. Fasc. Sci. Univ. Tokyo, Sec IA Math. 31(1985), 585 - 631. MR 86d:46039

[10] M. Sato, T. Kawai, M. Kashiwara, Microfunctions and Pseudo - differential Equations, Springer, Lect. Not. Math. 287 (1973), 265-529. MR 54:8747

Institute of Mathematics, University of Novi Sad, Trg D. Obradovića 4, 21000 Novi Sad, Yugoslavia 